\title{
VARIABILITÉ DES RÉGIMES PLUVIOMÉTRIQUES DANS LE NORD-OUEST DE L'ARGENTINE : PROBLĖMES POSÉS ET ANALYSE DURANT LA DEUXIÈME MOITIÉ DU VINGTIÈME SIĖCLE
}

\author{
O. PLANCHON ${ }^{1}$ et K. ROSIER ${ }^{2}$ \\ ${ }^{1}$ COSTEL LETG, UMR 6554 / FR 2116 CAREN-CNRS, Université Rennes-2 \\ olivier.planchon@uhb.fr \\ 2 DYMSET UMR 5064-CNRS, Université Bordeaux-3 karine_rosier@yahoo.fr
}

\section{Résumé}

La variabilité climatique, notamment pluviométrique, a été étudiée dans le NordOuest de l'Argentine durant la période 1951-2000. À une variabilité interannuelle d'autant plus forte que l'on se rapproche du piémont andin semi-aride et semi-aride (Mendoza, San Juán), s'ajoutent des oscillations de plus longue durée, en particulier une succession d'années anormalement arrosées à partir des années 1970. L'étude comparée de la période la plus arrosée (1971-1990) et de la période précédente, plus sèche (19511970), montre que l'augmentation des précipitations a été très variable d'une station à une autre, et l'analyse sur l'ensemble du vingtième siècle ne montre pas de lien significatif avec l'oscillation australe. Cependant, l'apparition plus fréquente de types de climats annuels plus humides, en particulier pour certaines stations situées en limite du domaine marqué par l'aridité (Santiago del estero), soulève le problème des conséquences économiques, humaines et environnementales d'un retour éventuel à une période moins arrosée.

Mots-clés : Variabilité climatique, régimes pluviométriques, Nord-Ouest argentin.

\section{Abstract \\ Variability of the precipitation regimes in the North-West of Argentina : some questions and analyses during the second part of the twentieth century.}

The climatic variability was studied in north-western Argentina for the period 19512000. The rainfall variability was especially analysed. North-western Argentina is a warm-temperate and subtropical area, which supplies agricultural produces not cultivated elsewhere in the country. In the subtropical latitudes of South America, the annual precipitation decreases between the wet Atlantic coast $(1000-2000 \mathrm{~mm} /$ year, without dry period) and the Pacific coast (Chile) almost without precipitation. The regional climatic divisions are essentially defined by the summer rainfall amount and by the duration of the summer rainy period. The Andes cordillera, the sub-Andean ranges and the Sierras Pampeanas cause an more complicated spatial distribution of precipitation. The sub-Andean ranges are exposed to the humid deviated trade winds blowing from the north-east and locally receive more than $1000 \mathrm{~mm} /$ year. The Province of Tucumán is called the "garden of the Argentine Republic", because of its varied agricultural produces. Further to the south, the Cuyo area (Provinces of Mendoza 
and San Juán) is subjected to an arid climate (100-200 mm / year), but vineyards and orchards have been developed with irrigation.

The inter-annual rainfall variability increases between the Pampa plain and the subAndean arid basins, but the northern humid subtropical sub-Andean slopes have more regular rainfall amounts. The climatic characteristics of north-western Argentina and the climatic annual characteristics of selected reference meteorological stations (precipitation regimes and frequency of the "annual climatic types") were shown by using the W. Köppen's classification. A period of abnormally rainy years was recorded in north-western Argentina between 1971 and 1990. The comparison between the rainy period 1971-1990 and the drier period 1951-1970 showed that the increase in precipitation was subjected to strong differences between the available meteorological stations. The analysis of the precipitation anomalies between 1901 and 2000 did not show significant correlations with the southern oscillation index. An other serie of rainy years occurred between 1917 and 1922, but the seventies - eighties rainy period was the longest of the twentieth century. Since the year 1991-1992, this rainy period is perhaps already ended in the area of Salta. The occurrence of several years with high positive precipitation anomalies during the period 1971-1990 was well shown for stations located on the spatial fringe of the arid and semi-arid areas. In these areas, the expansion and intensification of the agriculture benefited from a period of several years with higher rainfall amounts. Therefore, a return of years with drier climatic conditions could cause serious economic, human and environmental problems.

Keywords: Climatic variability, Precipitation regimes, North-West Argentina.

\section{Introduction}

Espace subtropical dans un pays à dominante tempérée, le Nord-Ouest de l'Argentine fournit à ce pays certaines productions agricoles non cultivées dans la plupart des autres régions de l'Argentine. Aux latitudes subtropicales de l'Amérique du Sud, le Nord-Ouest de l'Argentine s'insère dans un contexte climatique de large échelle qui implique un gradient pluviométrique est-ouest, de la bordure atlantique abondamment arrosée (entre 1000 et $2000 \mathrm{~mm}$ par an en moyenne) au littoral chilien quasiment sans pluie. Les nuances climatiques de ces régions reposent donc pour l'essentiel sur l'abondance et la durée de la saison pluvieuse d'été austral, mais sont aussi modulées par le relief diversifié et très contrasté de ce vaste espace (figure 1a).

Les plaines de l'est s'élèvent progressivement, vers l'ouest, jusqu'aux sierras Pampeanas (Sierra de Córdoba, de San Luis) et aux massifs préandins. Les sierras Pampeanas, orientées nord-sud, sont séparées par de vastes bassins fermés souvent occupés par des cuvettes salines. Plus à l'ouest, la cordillère des Andes porte les plus hauts sommets de la chaîne (Aconcagua, $6960 \mathrm{~m}$ ) et ses contreforts orientaux s'élargissent au nord de $27^{\circ} \mathrm{S}$, en direction de la Bolivie (Altiplano). Les reliefs de la cordillère des Andes et des chaînes pré-andines compliquent le gradient pluviométrique est-ouest en imposant des effets d'exposition et d'abri. Les contreforts abrupts des Andes exposés à l'est (Aconquija: $5500 \mathrm{~m}$, au-dessus de Tucumán) suscitent des précipitations 
abondantes (parfois plus de $1000 \mathrm{~mm}$ par an) dans les provinces de Salta et de Tucumán. Ces aires pluvieuses préandines se distinguent aussi par leur variabilité pluviométrique moindre que dans les régions qui les encadrent à l'est (Chaco) et à l'ouest (Altiplano). L'existence de ces climats régionaux abondamment arrosés est liée à des advections estivales d'air tropical humide dirigé par des flux de nord-est.
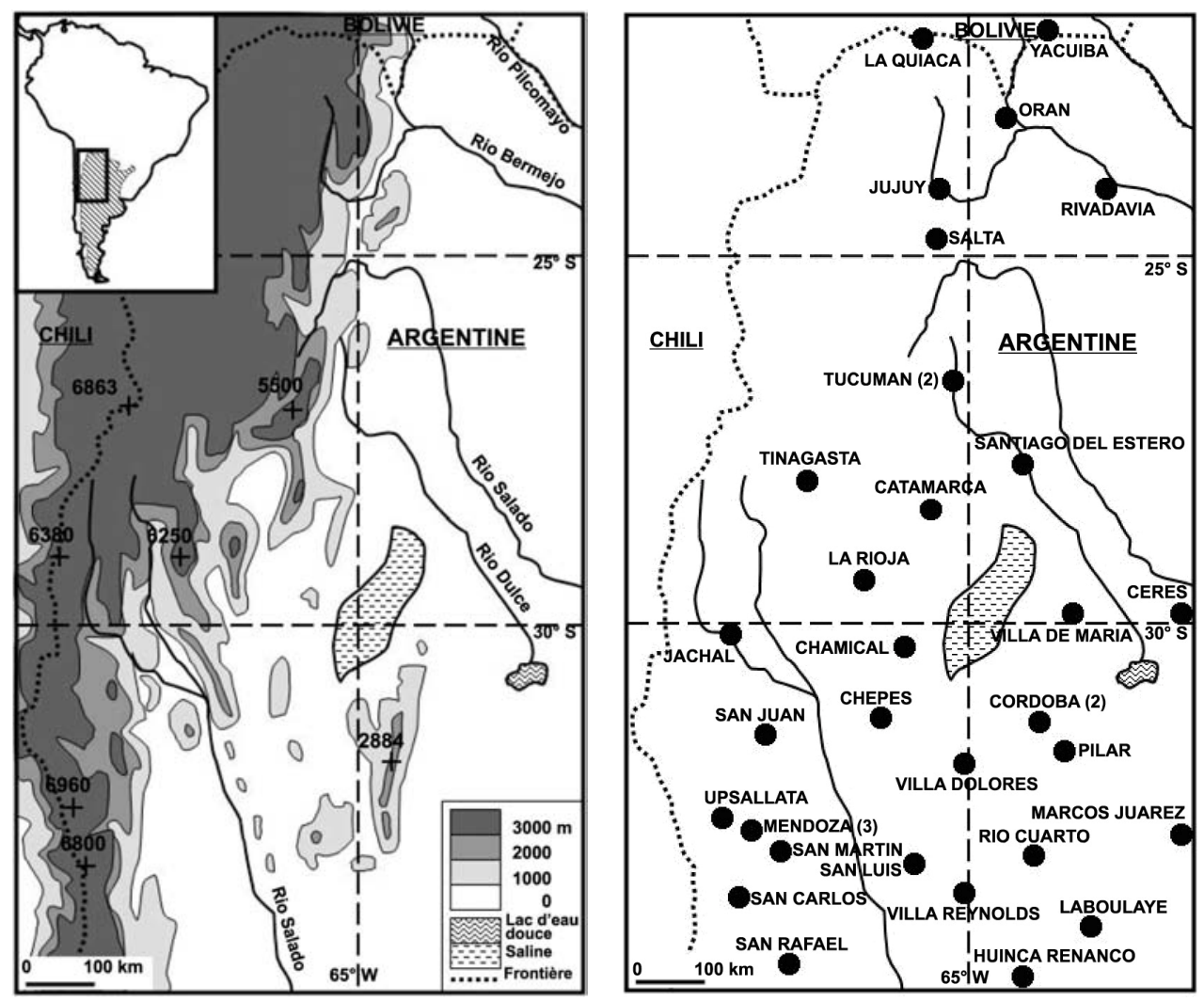

Figure 1 : Cartes de présentation de l'espace étudié. Maps of the studied area

A gauche : Relief et principaux cours d'eau du nord-ouest de l'Argentine. Localisation du Nord-Ouest argentin en Amérique du Sud (cadre en haut à gauche). Left : Relief and hydrography of North-West Argentina and location of the studied area in South America (box).

A droite : Localisation des stations des stations de référence (au moins deux stations à proximité d'une même agglomération : nombre de stations indiqué à Córdoba, Mendoza et Tucumán). Right : Location of the reference stations (at least two stations close to the same city : the number of stations is mentioned at Córdoba, Mendoza et Tucumán). 
Les pluies d'été se combinent à la chaleur estivale $\left(25^{\circ} \mathrm{C}\right.$ en janvier) pour faire de la Province de Tucumán le "Jardin de la République argentine" : canne à sucre, agrumes, soja et tabac. Plus au sud, le piémont de Cuyo autour de Mendoza est aride (100 à $200 \mathrm{~mm}$ par an en moyenne) avec quelques faibles pluies irrégulières, concentrées aussi sur l'été, mais grâce à l'irrigation vignobles et vergers se sont développés. Les impacts économiques de la variabilité climatique sont donc très sensibles dans ces régions et peuvent poser problème dans les projets d'aménagement (Hansen et al., 1996 ; United States Department of Agriculture, 2003 ; Salomon et Prat, 2004). Une extension de certaines cultures commerciales (agrumes et soja notamment) durant les dernières décennies du XXe siècle, dans des régions normalement "semiarides", ou, du moins, à période sèche prolongée d’hiver austral, a suscité des interrogations débouchant sur la nécessité d'établir un "état des lieux" climatique de cet espace en pleine mutation. Cette contribution, réalisée dans le cadre du GDR-2663 RICLIM (risques liés au climat), propose plusieurs approches de la variabilité pluviométrique, à l'aide de plusieurs méthodes, de façon à mettre en évidence les caractéristiques spatio-temporelles de cette variabilité.

\section{Données et méthodes}

Une classification climatique du Nord-Ouest argentin a été préalablement définie à l'aide des critères de la classification de W. Köppen, de façon à disposer d'une référence climatique de base avant d'étudier la variabilité interannuelle des quantités et des régimes pluviométriques. Les données, extraites du fichier GHCN-v2 (Global Historical Climatology Network : http://cdiac.esd.ornl.gov/ghcn/ghcn.html), ont été analysées pour la période 1951-2000 (34 stations en Argentine et une station frontalière en Bolivie : Yacuiba ; figure $1 \mathrm{~b}$ et tableau 1) en vue d'une cartographie des résultats. Les stations de Salta et de Mendoza ont pu être étudiées pendant 1901-2000. L'intérêt de ces deux stations est que la première (Salta) est représentative du milieu très particulier du piémont andin du Nord-Ouest argentin et des yungas (forêts subtropicales et tropicales du versant oriental des Andes), à saisons pluviométriquement contrastées (précipitations abondantes en été), tandis que la seconde (Mendoza), au pied des Andes aussi mais plus au sud, est représentative d'un vaste espace marqué par l'aridité. La saison pluvieuse étant à cheval sur le changement d'année civile, la succession des mois de l'année a été inversée : le premier mois de l'année est juillet au lieu de janvier. Les premières années des séries analysées sont donc 1951-1952 et 1901-1902. 


\begin{tabular}{|l|c|c|r|r|r|}
\hline \multicolumn{1}{|c|}{ STATION } & LAT. $(\mathrm{S})$ & LON. $(\mathrm{W})$ & $\begin{array}{c}\text { ALT. } \\
(\mathrm{m})\end{array}$ & $\% \mathrm{MQ}(\mathrm{P})$ & $\% \mathrm{MQ}(\mathrm{T})$ \\
\hline YACUIBA (Bolivie) & 22.00 & 63.70 & 580 & 34.5 & $/$ \\
\hline LA QUIACA OBSERVATORIO & 22.10 & 65.60 & 3462 & 22.0 & 17.5 \\
\hline ORÁN AEROPUERTO & 23.15 & 64.32 & 357 & 38.2 & $/$ \\
\hline JUJUY AEROPUERTO & 24.38 & 65.08 & 921 & 15.3 & 27.5 \\
\hline SALTA AEROPUERTO & 24.85 & 65.48 & 1238 & 1.3 & 5.0 \\
\hline RIVADAVIA & 24.17 & 62.90 & 204 & 25.2 & 27.3 \\
\hline TUCUMÁN AEROPUERTO & 26.85 & 65.10 & 421 & 25.5 & 47.7 \\
\hline TUCUMÁN OBSERVATORIO & 26.80 & 65.20 & 481 & 51.3 & 52.7 \\
\hline SANTIAGO DEL ESTERO & 27.77 & 64.30 & 198 & 9.5 & 13.0 \\
\hline TINOGASTA & 28.07 & 67.57 & 1201 & 59.0 & $/$ \\
\hline LA RIOJA AEROPUERTO & 29.38 & 66.82 & 438 & 6.8 & 8.3 \\
\hline CATAMARCA AEROPUERTO & 28.60 & 65.77 & 454 & 8.7 & 9.3 \\
\hline VILLA DE MARÍA & 29.90 & 63.70 & 341 & 31.7 & $/$ \\
\hline CERES AEROPUERTO & 29.88 & 61.95 & 87 & 1.2 & 5.3 \\
\hline JÁCHAL & 30.25 & 68.75 & 1164 & 25.8 & 67.8 \\
\hline SAN JUÁN AEROPUERTO & 31.57 & 68.42 & 597 & 5.7 & 7.7 \\
\hline CHAMICAL AEROPUERTO & 30.40 & 66.30 & 461 & 19.2 & $/$ \\
\hline CHEPES & 31.33 & 66.60 & 657 & 32.8 & 34.2 \\
\hline VILLA DOLORES AEROPUERTO & 31.95 & 65.13 & 569 & 34.3 & $/$ \\
\hline CÓRDOBA AEROPUERTO & 31.32 & 64.22 & 484 & 5.5 & 6.7 \\
\hline CÓRDOBA & 31.40 & 64.20 & 425 & 15.5 & 31.3 \\
\hline PILAR OBSERVATORIO & 31.67 & 63.88 & 338 & 7.3 & 8.2 \\
\hline UPSALLATA & 32.60 & 69.33 & 1891 & 59.2 & $/$ \\
\hline SAN CARLOS (MZA) & 33.77 & 69.03 & 940 & 46.5 & $/$ \\
\hline SAN MARTÍN (MZA) & 33.08 & 68.42 & 653 & 37.8 & $/$ \\
\hline MENDOZA AEROPUERTO & 32.83 & 68.78 & 705 & 4.2 & 9.3 \\
\hline CHACRAS DE CORIA & 32.98 & 68.86 & 921 & 42.3 & $/$ \\
\hline MENDOZA OBSERVATORIO & 32.90 & 68.80 & 828 & 13.5 & 31.5 \\
\hline SAN LUÍS AEROPUERTO & 33.27 & 66.35 & 710 & 1.5 & 7.2 \\
\hline VILLA REYNOLDS AEROPUERTO & 33.73 & 65.38 & 485 & 24.8 & 48.3 \\
\hline RÍO CUARTO AEROPUERTO & 33.12 & 64.23 & 420 & 4.3 & 8.3 \\
\hline MARCOS JUÁREZ AEROPUERTO & 32.70 & 62.15 & 110 & 31.5 & $/$ \\
\hline SAN RAFAEL AEROPUERTO & 34.58 & 68.40 & 745 & 22.5 & 25.3 \\
\hline HUINCA RENANCÓ & 34.83 & 64.27 & 190 & 12.0 & $/$ \\
\hline LABOULAYE AEROPUERTO & 34.13 & 63.37 & 136 & 23.3 & 25.3 \\
\hline
\end{tabular}

Tableau 1 : Liste des stations météorologiques de référence et coordonnées géographiques (LAT. latitude en dixièmes de degrés ; LON. Longitude en dixièmes de degrés ; ALT. Altitude en $\mathrm{m}$ ). Pourcentage de données manquantes entre 1951 et 2000 : précipitations (\% MQ (P)) et températures (\% MQ (T) ; / pas de données). List of the meteorological stations and geographical coordinates (LAT. Latitude in tenths of degrees ; LON. Longitude in tenths of degrees ; ALT. Altitude in metres). Percentage of missing values between 1951 and 2000 : precipitation (\% MQ (P)) and temperature (\% MQ (T) ; / no data).

Source : GHCN (Global Historical Climatology Network). Fichier de données développé et diffusé par CDIAC / ORNL (Carbon Dioxide Information Analysis Center / Oak Ridge National Laboratory) et NCDC (National Climatic Data Center). http://cdiac.esd.ornl.gov/ghcn/ghcn.html. Data source: GHCN (Global Historical Climatology Network). Data file prepared and published by CDIAC / ORNL (Carbon Dioxide Information Analysis Center / Oak Ridge National Laboratory) et NCDC (National Climatic Data Center). http://cdiac.esd.ornl.gov/ghcn/ghcn.html. 
Certaines séries de données ont été obtenues pour deux voire trois stations situées à proximité d'une même localité (Tucumán, Córdoba, Mendoza). Compte tenu des données manquantes, ces quelques groupes de stations voisines les unes des autres ont permis de comparer les résultats obtenus avec chacun des fichiers. La qualité des données étant très inégale d'une station à l'autre, une partie seulement des stations disponibles a pu faire l'objet de toutes les analyses effectuées pour la préparation de cet article. Des tableaux plus détaillées concernant ces stations seront présentés ultérieurement dans l'article. Les régimes pluviométriques de l'espace étudié ont été définis d'après la classification de W. Köppen, en raison de l'intérêt de cette classification pour la définition de seuils de sécheresse et d'aridité. W. Köppen a, en effet, borné l'aridité par comparaison entre précipitations et température, en introduisant des nuances selon les régimes pluviométriques et thermiques. Les tables et méthodes permettant de calculer les seuils limites de semi-aridité et d'aridité, d'une part (pas de temps annuel) et de sécheresse d'autre part (pas de temps mensuel), sont données ci-dessous :

\begin{tabular}{|r|r|l|}
\hline $\begin{array}{l}T^{\circ} \mathrm{C} \text { moyenne } \\
\text { annuelle }\end{array}$ & $\begin{array}{l}\text { Pmm annuelles : } \\
\text { seuil «BS » }\end{array}$ & $\begin{array}{l}\text { Pmm annuelles } \\
\text { seuil «BW }\end{array}$ \\
\hline 30 & 800 & 400 \\
\hline 25 & 700 & 350 \\
\hline 20 & 600 & 300 \\
\hline 15 & 500 & 250 \\
\hline 10 & 400 & 200 \\
\hline 5 & 300 & 150 \\
\hline 0 & 200 & 100 \\
\hline-5 & 100 & 50 \\
\hline
\end{tabular}

Tableau 2 : Identification des climats semi-arides (BS) et arides (BW), selon la classification de W. Köppen. Identification of semi-arid (BS) and arid (BW) climates, according to the W. Köppen's classification.

À une valeur de la température moyenne annuelle donnée correspondent deux seuils pluviométriques au-dessous desquels le climat est classé "BS" (semi-aride) ou "BW" (aride). En dehors des climats arides ou semi-arides et de la zone chaude, la sécheresse saisonnière a été définie en fonction du régime pluviométrique. Une saison sèche d'hiver (climats "Cw") est définie si le mois le moins arrosé d'hiver reçoit moins de 1/10 des précipitations du mois le plus arrosé d'été. Sinon, le climat est classé Cf (pas de saison sèche), ou Cs (Saison sèche d'été) si le mois le moins arrosé d'été reçoit moins de $1 / 3$ des précipitations du mois le plus arrosé d'hiver. Un correctif a été apporté par la suite : le climat est classé Cs si les précipitations moyennes du mois le moins arrosé de l'été, exprimées en mm, sont inférieures au double de la température du même mois exprimée en ${ }^{\circ} \mathrm{C}$. Un régime est dit "à période sèche" lorsqu'au moins un mois dans l'année est considéré comme sec, au sens de la classification de W. Köppen. Afin de détecter d'éventuelles modifications des conditions climatiques durant la deuxième moitié du vingtième siècle, la 
classification a été appliquée à deux sous périodes de 20 ans (1951-1970 et 1971-1990, périodes retenues après avoir calculé les moyennes de précipitations glissantes pour les périodes 1951-1970, 1961-1980, 1971-1990 et 1981-2000), mais aussi année par année, de façon à déterminer des "types de climats annuels" et la fréquence d'apparition de chacun de ces types. "Type de climat annuel" est un terme naturellement impropre à la définition même de "climat", mais déjà employé (Brisse et al., 1982), pour définir l'ambiance climatique d'une année donnée. Enfin, à l'aide des séries séculaires de Salta et de Mendoza, l'étude de la variabilité climatique dans le Nord-Ouest argentin a permis la recherche d'autres à plus long terme (vingtième siècle), mais aussi à la recherche de connexions avec l'oscillation australe.

\section{Trame et gradients climatiques : aridité et pluies d'été}

En Amérique du Sud, les régions arides (BW) sont confinées à l'ouest du continent, de part et d'autre de la cordillère des Andes ; l'aridité quasi absolue étant limitée à la bordure pacifique (Pérou et Chili septentrional). En revanche, au centre et à l'est du continent, les climats des Pampas (Cf et $\mathrm{Cw}$ ) sont directement en contact, au nord (Brésil, Paraguay), avec le domaine tropical humide (Af) ou à saison des pluies bien marquée (Aw). Pour rappel, les latitudes subtropicales du continent sud-américain sont caractérisées par un gradient pluviométrique est-ouest, de la bordure atlantique abondamment arrosée (entre 1000 et $2000 \mathrm{~mm}$ par an en moyenne) au littoral chilien quasiment sans pluie (moins de $10 \mathrm{~mm}$ par an au nord de $26^{\circ} \mathrm{S}$ ). Dans le nord de l'Argentine, les précipitations liées aux masses d'air d'origine atlantique s'amoindrissent vers l'intérieur, en direction des Andes. Ainsi, vers $30^{\circ} \mathrm{S}$, l'aridité affecte déjà le versant argentin de la cordillère, particularité climatique déjà soulignée par E. de Martonne (1935). De plus, le zonda (vent de type föhn), déjà sensible à Mendoza $\left(33^{\circ} \mathrm{S}\right)$, aggrave l'aridité du piémont de Cuyo qu'il balaie périodiquement (Endlicher, 1998). Plus au nord, les précipitations annuelles diminuent progressivement de la bordure atlantique vers l'intérieur du continent, atteignant, dans le Chaco, un minimum de 500 à $700 \mathrm{~mm}$ aux confins de l'Argentine, du Paraguay et de la Bolivie (climat de type BS : Planchon, 2003). Mais, plus à l'ouest, le rebord abrupt des massifs pré-andins et de l'altiplano (Aconquija, $5500 \mathrm{~m}$ ), suscite, par ascendance orographique, des précipitations nettement plus abondantes sur le piémont et les versants exposés à l'est et au nord-est (alizés), d'où la présence des yungas. Les deux stations de Tucumán reçoivent plus de $1000 \mathrm{~mm}$ par an (climat de type Cw), tandis que la puna (steppe de haute altitude) est semi-aride (La Quiaca, 1951-1995 : 327 $\mathrm{mm})$. 


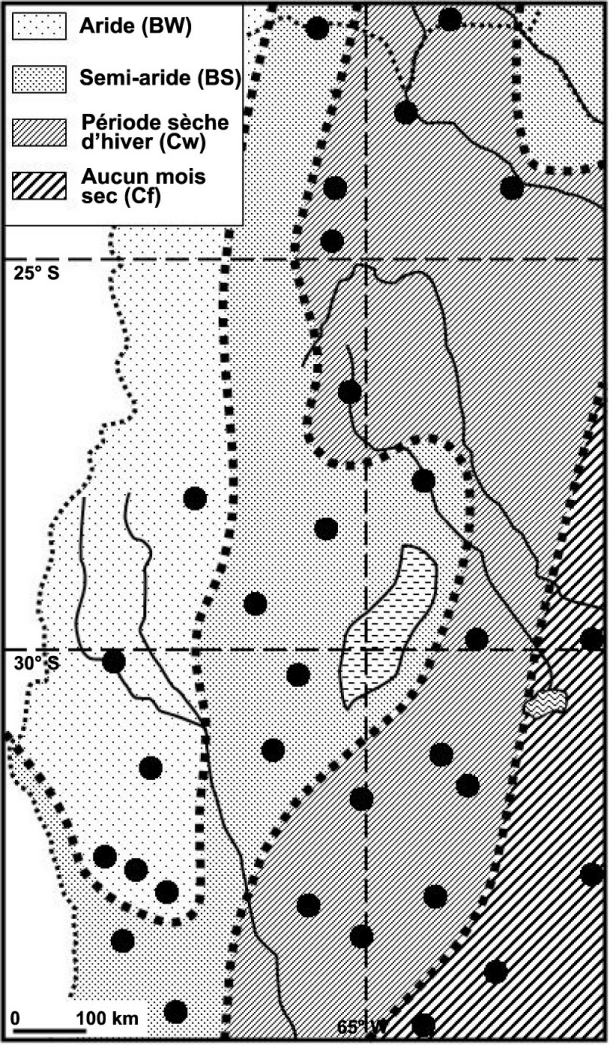

Figure 2 : Types de climats dans le NordOuest de l'Argentine, d'après la classification de W. Köppen (période 19512000). Types of climates in the North-West of Argentina, according to the W. Köppen's classification (period 1951-2000).

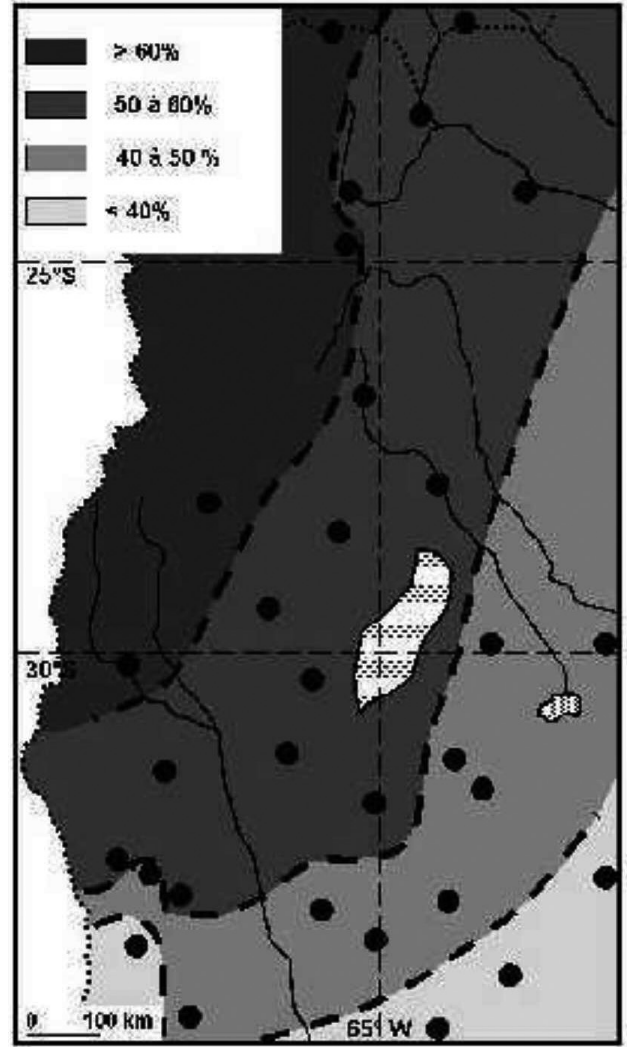

Figure 3 : Part des précipitations estivales (DJF) par rapport au total annuel, en pourcentage (période 1951-2000). Percentage of the summer precipitation (DJF) in proportion to the annual amount (period 1951-2000).

L'apparition, l'intensification et l'allongement progressif d'une période "sèche" hivernale accompagnée par l'amoindrissement de la saison pluvieuse se concentrant sur l'été crée, au sud de l'espace étudié, un gradient climatique depuis la Pampa humide jusqu'à l'aridité du piémont andin abrité de Cuyo, en passant successivement par les climats de type Cf, Cw, BS et BW (figure 2). On retrouve ce gradient et cette trame climatique, fondée sur les rythmes saisonniers incluant la durée et l'intensité de la sécheresse, avec la classification des climats mondiaux de N. Creutzburg (1950) décrite et commentée par J. Blüthgen (1964), puis celle de C. Troll et K.H. Paffen (1964) et celle de W. Lauer et P. Frankenberg (1992), décrites et commentées par W. Lauer (1993). 


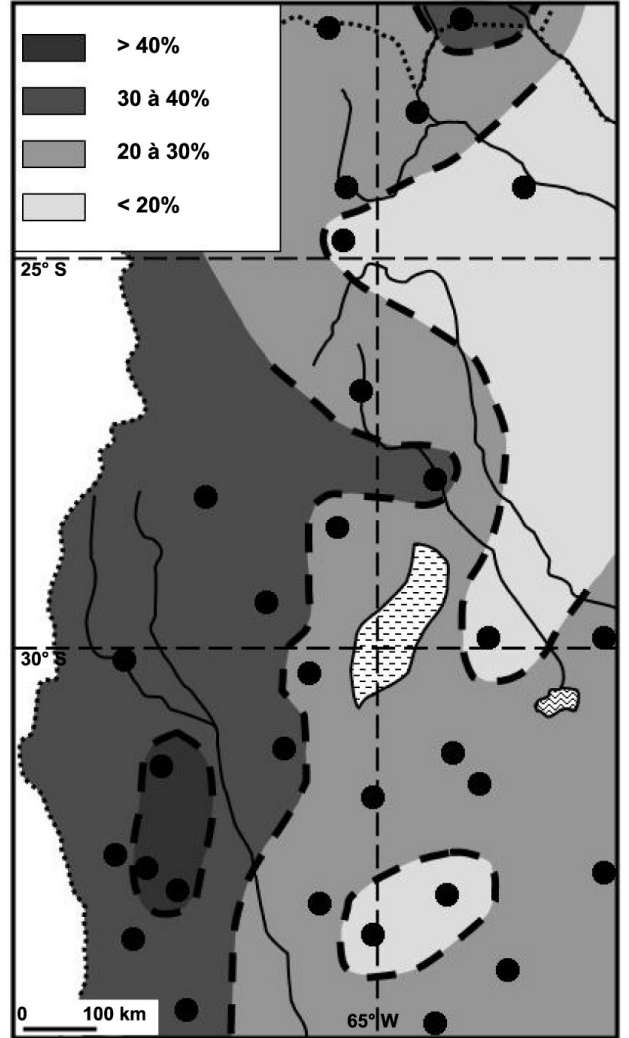

Figure 4 : Variabilité des précipitations annuelles (1951-2000), exprimée en coefficients de variation. Variability of the annual precipitation (1951-2000), expressed in coefficients of variation.

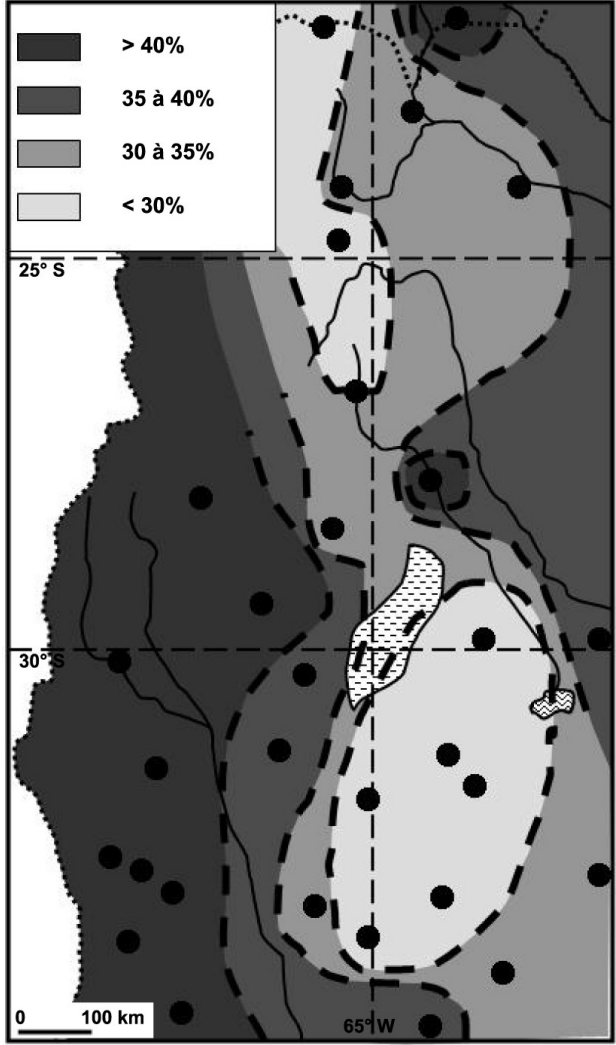

Figure 5 : Variabilité des précipitations estivales (DJF ; période 1951-2000), exprimée en coefficients de variation. Variability of the summer precipitation (DJF ; périod 1951-2000), expressed in coefficients of variation.

L'été est la saison la plus arrosée sur l'ensemble de l'espace étudié et la part des pluies d'été par rapport aux précipitations annuelles se renforce en direction de l'ouest et surtout du nord-ouest (figure 3). Les totaux pluviométriques annuels sont donc plus directement liés à l'abondance des pluies d'été (été : D-JF) dans les climats marqués par l'aridité. Ainsi, la station de La Quiaca sur l'Altiplano reçoit $68 \%$ de ses précipitations annuelles de décembre à février. C'est l'abondance de la période pluvieuse de saison chaude et sa durée qui permet de déterminer une gradation climatique longitudinale et de différencier les climats à l'échelle régionale. D'est en ouest, l'été reste la saison la plus arrosée grâce à la prédominance d'averses orageuses convectives, en connexion avec les advections d'air tropical humide sur la Pampa. Au-delà des sierras Pampeanas, les pluies d'été sont faibles et irrégulières, mais représentent plus de la moitié du total annuel sur la majorité des stations. Les contreforts andins des 
régions septentrionales de l'espace étudié font obstacle aux advections estivales d'air tropical humide dirigé par les flux dominants de nord-est (Prohaska, 1976) ; ces ascendances orographiques accentuent la convection. En été austral, les basses pressions thermiques de surface situées au-dessus du Chaco attirent les alizés boréaux, déviés en direction du centre de l'Amérique du Sud, d'où l'appellation de "mousson sud-américaine" employée par certains auteurs (Zhou et Lau, 1998). La déviation des alizés par la cordillère des Andes favorise leur intervention en direction du Nord-Ouest argentin. C'est donc un régime pluviométriquement contrasté à pluies d'été abondantes, annonciateur des régimes tropicaux des espaces situés plus au nord, qui domine sur le piémont andin septentrional, et entretient des formations végétales spécifiques telles que les yungas. Le gradient climatique avec l'Altiplano semi-aride en est d'autant resserré et brutal.

Les climats marqués par l'aridité subissent aussi la variabilité pluviométrique interannuelle la plus forte (piémont de Cuyo : région de Mendoza et de San Juán, figure 4). La variabilité des précipitations estivales est donc aussi logiquement la plus forte sur les stations subandines arides et semi-arides, leurs faibles précipitations étant concentrées sur les mois d'été (figure 5). Inversement, les stations subandines septentrionales abondamment arrosées en été ont une variabilité moindre, Salta notamment $(23,4 \%)$, ce qui souligne l'importance des faits circulatoires estivaux (advections d'air tropical humide) dans l'existence de ce climat régional à caractère subtropical. La station de La Quiaca, semi-aride mais de latitude tropicale bénéficie d'une variabilité relativement faible (moins de 30 \% pour l'année ; moins de 25 \% pour l'été).

\section{Une anomalie pluviométrique majeure a-t-elle eu lieu à la fin du $\mathrm{XX}^{\mathrm{e}}$ siècle?}

Les fluctuations climatiques, surtout pluviométriques, sont importantes à moyen terme, et les régions subtropicales du Nord-Ouest argentin sont soumises à une alternance de périodes humides et de périodes sèches (Gonzalez Loyarte, 1995 ; Salomon et Prat, 2004). Le dernier quart du $\mathrm{XX}^{e}$ siècle a ainsi été caractérisé par une abondance pluviométrique plus importante que durant les décennies précédentes. M.C. Prat et J.M. Sayago (2003) ont notamment souligné l'abondance pluviométrique des trente dernières années du vingtième siècle à Tucumán, sensible surtout dans les régions subtropicales du Nord-Ouest argentin. La deuxième moitié du vingtième siècle incite donc à une étude comparée des deux périodes 1951-1970 et 1971-1990, cette dernière incluant le mieux, compte tenu des données disponibles, la période "pluvieuse" (tableau 3). 


\begin{tabular}{|l|c|c|c|c|c|c|c|}
\hline \multirow{2}{*}{ STATION } & \multicolumn{2}{|c|}{ Précipitations moyennes annuelles } & Ecart (\%) & MQ (\%) & MQ (\%) \\
\cline { 2 - 7 } & $1951-70$ & $1961-80$ & $1971-90$ & $1981-00$ & $81-00 / 71-90$ & $1951-2000$ & $91-00 / 51-00$ \\
\hline SALTA AERO & 691.0 & 726.9 & $\mathbf{7 8 9 . 7}$ & 749.6 & -5.1 & 1.3 & 75.0 \\
\hline $\begin{array}{l}\text { SANTIAGO DEL } \\
\text { ESTERO }\end{array}$ & 512.6 & 616.6 & $\mathbf{6 4 7 . 5}$ & 573.2 & -11.5 & 9.5 & 96.5 \\
\hline LA RIOJA AERO & 315.2 & 339.9 & 389.2 & $\mathbf{3 9 4 . 0}$ & +1.2 & 6.8 & 58.5 \\
\hline CATAMARCA AERO & 383.1 & 437.6 & $\mathbf{4 7 6 . 7}$ & 418.7 & -12.2 & 8.7 & 59.6 \\
\hline CERES AERO & 831.6 & 901.3 & $\mathbf{9 7 4 . 9}$ & 960.2 & -1.5 & 1.2 & 71.4 \\
\hline SAN JUÁN AERO & 88.2 & 91.7 & 99.2 & $\mathbf{1 2 3 . 2}$ & +24.2 & 5.7 & 44.1 \\
\hline CÓRDOBA AERO & 762.0 & 819.7 & 863.5 & $\mathbf{8 6 6 . 2}$ & +0.3 & 5.5 & 42.4 \\
\hline PILAR OBS. & 713.6 & 772.8 & $\mathbf{8 2 4 . 7}$ & 774.2 & -6.1 & 7.3 & 65.9 \\
\hline MENDOZA AERO & 165.5 & 177.6 & 205.3 & $\mathbf{2 2 3 . 8}$ & +9.0 & 4.2 & 84.0 \\
\hline SAN LUÍS AERO & 550.0 & 583.2 & $\mathbf{6 2 6 . 8}$ & 607.6 & -3.1 & 1.5 & 88.9 \\
\hline RÍO CUARTO AERO & 777.5 & 814.0 & 864.4 & $\mathbf{9 0 9 . 6}$ & +5.2 & 4.3 & 100.0 \\
\hline
\end{tabular}

Tableau 3 : Moyennes des précipitations annuelles calculées pour des périodes glissantes de vingt ans, entre 1951 et 2000. En italique : moyennes des périodes les moins arrosées ; en gras sur fond gris : moyennes des périodes les plus arrosées. Ecart (\%) 81-00/71-90 : écart pluviométrique annuel (en \%) de la période 1981-2000 par rapport à la période 1971-1990. MQ (\%) 1951-2000 : Pourcentage de données manquantes pour la période 1951-2000. MQ (\%) 91-00/51-00 : pourcentage de données manquantes de la période 1991-2000 par rapport à la période 1951-2000. Mean annual precipitation for sliding periods of twenty years, between 1951 and 2000. In italic characters : mean annual precipitation for the less rainy periods; in bold characters (grey boxes) : mean annual precipitation for the rainiest periods. Ecart (\%) 81-00/7190 : Difference (\%) in annual precipitation between the period 1981-2000 and the period 1971-1990. MQ (\%) 1951-2000 : percentage of missing values during the period 1951-2000. MQ (\%) 91-00/51-00 : percentage of missing values during the period 1991-2000 in the period 1951-2000.

Le tableau 3 présente les 11 stations pour lesquelles les données pluviométriques ont été disponibles pour la période 1951-2000 avec le plus faible pourcentage de données manquantes (moins de $10 \%$ du total). Quatre moyennes glissantes de 20 ans ont pu être calculées, permettant de cerner le mieux possible la période pluvieuse étudiée. Alors que la période 1951-1970 a été partout la moins arrosée, la plus arrosée se partageait (avec des écarts le plus souvent peu sensibles) entre 1971-1990 et 1981-2000. Cependant, l'été (D-J-F) a été plus arrosé en 1971-1990 à La Rioja, Mendoza et Córdoba, mais la décennie 1991-2000 pose problème quant à la fiabilité des résultats, puisque les données manquantes se concentrent durant cette décennie. Il a donc été préférable de comparer les deux périodes 1951-1970 et 1971-1990, cette dernière étant sélectionnée pour représenter la période la plus arrosée. 


\subsection{Sensibilité des "contacts climatiques" : mise à l'épreuve par les types de climats annuels}

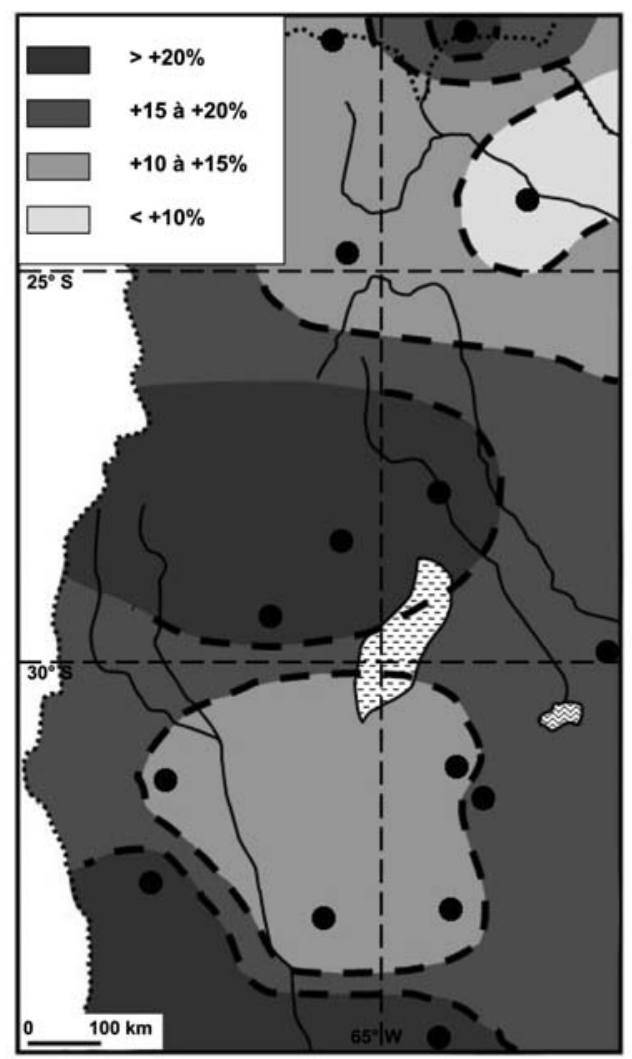

Figure 6 : Ecart pluviométrique annuel (en \%) de la période 1971-1990 par rapport à la période 1951-1970. Difference (\%) in annual precipitation between the period 1971-1990 and the period 1951-1970.

L'augmentation des précipitations d'une période à l'autre a été très variable selon les stations (figure 6) : pas de variation à Rivadavia (+ 0,15 \%) mais $+23,5$ à + 26,3 \% sur les stations subandines semi-arides situées au centre de l'espace étudié (Catamarca, La Rioja et Santiago del Estero). Les stations normalement les plus arrosées ont, pour l'essentiel, connu de plus faibles hausses, notamment les stations de la pampa au sud-est (Río Cuarto + 11,2 \%). Il est difficile de dégager une logique spatiale de ce résultat, compte-tenu de certaines différences observées d'une station à l'autre, mais aussi de la faible densité du réseau de stations sur ce vaste espace à la topographie contrastée. La valeur de l'augmentation des précipitations est somme toute peu importante sur l'ensemble de la période de vingt ans prise en compte, ce qui souligne le poids de la variabilité interannuelle. Autre méthode pour appréhender la variabilité climatique interannuelle, la fréquence des types de climats annuels. Elle apporte 
des informations supplémentaires par rapport à la seule prise en compte des moyennes, pour l'ensemble de la période étudiée et les deux sous-périodes comparées (tableau 4).

\begin{tabular}{|c|c|c|c|c|c|c|c|}
\cline { 3 - 8 } \multicolumn{1}{c|}{} & Type de & \multicolumn{5}{c|}{ Type de climat annuel dominant } \\
\cline { 3 - 8 } & \begin{tabular}{c} 
climat \\
\cline { 3 - 8 } \multicolumn{1}{c|}{}
\end{tabular} & \multicolumn{2}{|c|}{$1951-1990$} & \multicolumn{2}{c|}{$1951-1970$} & \multicolumn{2}{c|}{$1971-1990$} \\
\hline Station & Köppen & Köppen & Freq. \% & Köppen & Freq. \% & Köppen & Freq. \% \\
\hline Catamarca & BS & BS & 80,0 & BS & 75,0 & BS & 85,0 \\
\hline Ceres & Cf & Cw & 85,0 & Cw & 85,0 & Cw & 85,0 \\
\hline Córdoba & Cw & Cw & 100,0 & Cw & 100,0 & Cw & 100,0 \\
\hline La Quiaca & BS & BS & 75,0 & BS & 75,0 & BS & 75,0 \\
\hline La Rioja & BS & BS & 67,5 & BS/BW & 50,0 & BS & 85,0 \\
\hline Mendoza & BW & BW & 87,5 & BW & 95,0 & BW & 80,0 \\
\hline Río Cuarto & Cw & Cw & 95,0 & Cw & 90,0 & Cw & 100,0 \\
\hline Rivadavia & Cw & Cw & 52,5 & Cw & 55,0 & Cw/BS & 50,0 \\
\hline Salta & Cw & Cw & 97,5 & Cw & 95,0 & Cw & 100,0 \\
\hline San Juán & BW & BW & 100,0 & BW & 100,0 & BW & 100,0 \\
\hline San Luís & Cw & Cw & 62,5 & Cw & 55,0 & Cw & 70,0 \\
\hline Santiago & BS & BS & 60,0 & BS & 85,0 & Cw & 60,0 \\
\hline
\end{tabular}

Tableau 4 : Fréquence (Freq., en pourcentage) des types de climats annuels "dominants" (définis selon la classification de W. Köppen) pour quelques stations du Nord-Ouest argentin. Frequency (Freq., in percentages) of the prevailing annual climatic types (according to the W. Köppen's classification), for some stations in NorthWestern Argentina.

Le climat de toutes les stations, à l'exception d'une seule (Ceres), est aussi le climat "dominant", le plus fréquemment (et majoritairement) observé d'une année à l'autre sur l'ensemble des séries. À Ceres, les années de type "Cw" sont très largement majoritaires, alors que le climat de cette station est de type "Cf". Ici, la moyenne pose donc problème quand à sa signification. La variabilité pluviométrique hivernale étant très forte dans cette région (Planchon, 1997), la quasi-totalité des années présente un régime suffisamment contrasté pour un classement en "Cw", mais le ou les mois concernés ne sont pas toujours les mêmes d'une année à l'autre. La moyenne pondère le régime pluviométrique en atténuant les contrastes saisonniers et en créant un régime compensé. Cette station présente même une année à régime "s" (mois sec(s) en été), régime qui est sensé ne pas exister (en moyenne) dans cette partie de l'Amérique du Sud. La fréquence des types de climats annuels à Rivadavia, dans le Chaco, reflète bien la position de cette station en la "limite" des types "Cw" et "BS", puisque ces deux types de climat y étaient à peu près également représentés durant la période de référence. Le léger avantage du type "Cw" (52,5 \%) confirme l'appartenance de Rivadavia à ce dernier type de climat, en se référant à la moyenne. Les stations les plus représentatives du climat auquel elles sont censées appartenir sont bien visibles : à Córdoba et à San Juán, toutes les années 
ont été classées respectivement "Cw" et "BW". À Salta, seule une année a été classée différemment (BS) du type de climat de cette station (Cw). La fréquence des types de climats annuels rend compte aussi du gradient climatique spatial conduisant à l'aridité du piémont andin de Cuyo : de Santiago del estero à San Juán, au pied des Andes et de Río Cuarto à Mendoza, à travers la région des sierras Pampeanas. D'une station à l'autre, la diminution de la part des années de type "Cw" (puis leur disparition), le relais pris par les années de type "BS", puis l'importance prise par les années de type "BW" illustrent, mieux encore que la carte climatique moyenne, comment se module le gradient climatique, en prenant en compte les types de climats annuels.

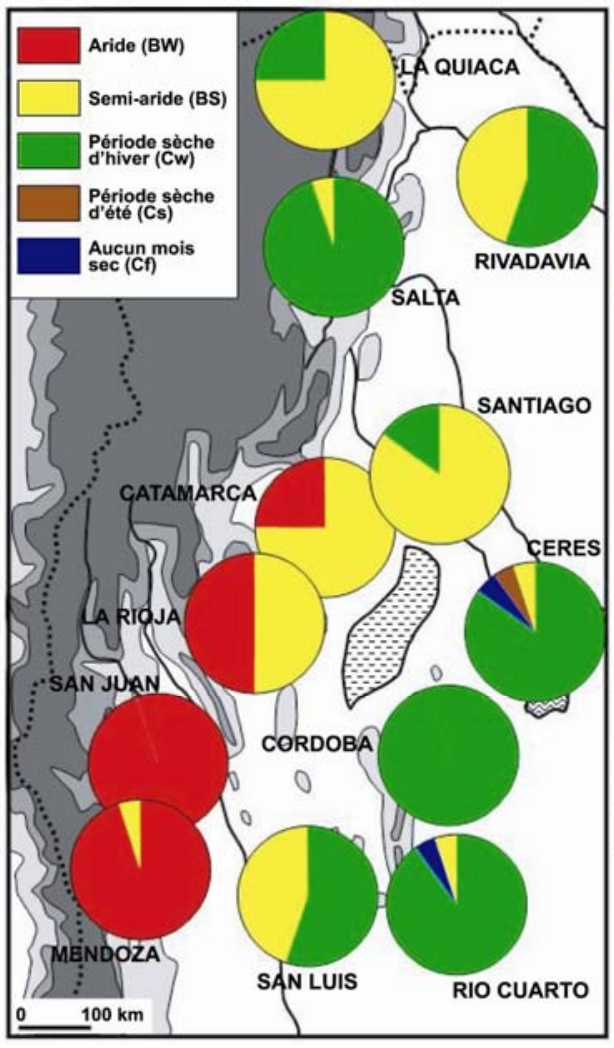

a) période 1951-70

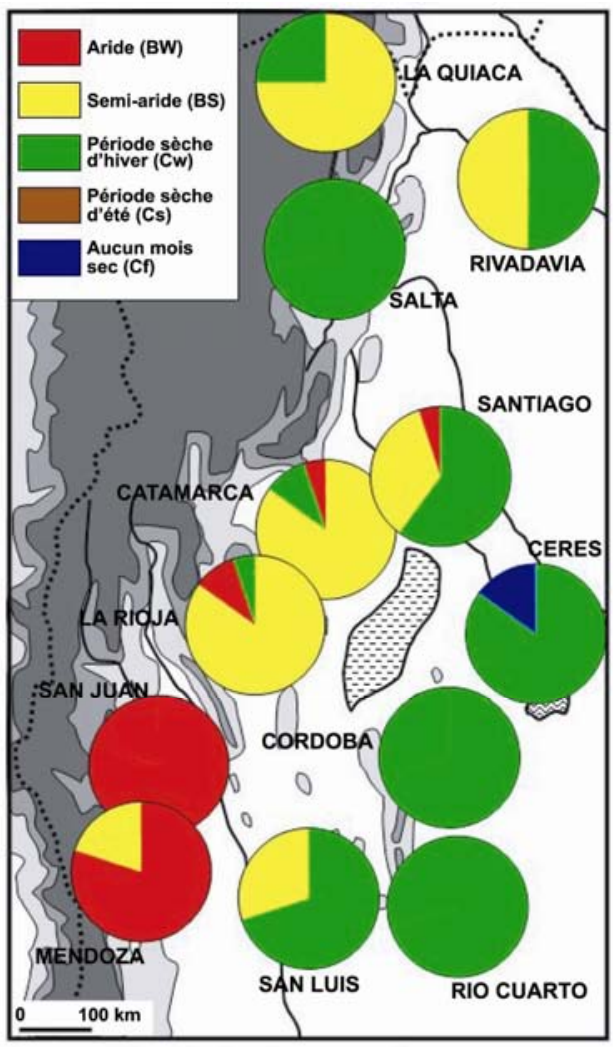

b) période 1971-90

Figure 7 : Fréquence des types de climats annuels, d'après la classification de W. Köppen. Fond de carte : relief et hydrographie. Frequency of the annual climatic types, according to the W. Köppen's classification (period (1951-1970). Pie charts on the map of relief and hydrography.

Par rapport à l'ensemble de la période 1951-1990, les types de climats annuels dominants le sont toujours, station par station, pour les deux "souspériodes" 1951-1970 et 1971-1990 (figures 7a et 7b), sauf à Santiago del estero. À Santiago, le type "Cw" est devenu dominant en 1971-1990, traduisant ainsi 
un plus grand nombre d'années à saison pluvieuse abondante que durant la période précédente (1951-1970 : 3 années "Cw" ; 1971-1990 : 12 années "Cw"). À Catamarca et La Rioja sont apparus des types de climats annuels plus arrosés (Cw) qui n'existaient pas durant la période 1951-1970. La fréquence plus élevée des types de climats annuels plus arrosés que le type de climat "moyen" de la station, caractérise la quasi totalité des stations de référence, surtout les stations subandines marquées par l'aridité (Santiago, Catamarca et La Rioja). Leur situation de transition ou de contact climatique les rend d'autant plus sensibles à la moindre anomalie. La période 1971-1990 semble bien avoir connu davantage d'années à types de climats plus arrosés que la période de 20 ans précédente. Cependant, non seulement les types de climats annuels dominants sont pour l'essentiel inchangés entre les deux périodes, mais aussi, l'apparition plus fréquente d'années "arrosées" a été inégalement ressentie selon les stations, cet aspect faisant ressortir les stations des marges semi-arides. Un examen plus détaillé s’impose, année par année, mais aussi sur une série plus longue.

\subsection{Existe-t-il un lien entre la variabilité pluviométrique du Nord-Ouest argentin et l'oscillation australe ?}

L'examen des anomalies pluviométriques, année par année, pour les stations de Mendoza (piémont aride de Cuyo) et de Salta (contreforts andins subtropicaux), dont les séries de données disponibles par le fichier GHCN sont les plus complètes au vingtième siècle, fait ressortir une succession d'années anormalement arrosées durant les décennies 70 et 80, avec des intensités inégales d'une année à l'autre. La prise en compte des anomalies sur la plus grande partie du siècle permet d'avoir un recul qui fait d'autant mieux ressortir l'originalité pluviométrique de ces deux décennies (tableau 5). Quelques données manquantes à Mendoza n'ont pas permis de réaliser le tableau jusqu'aux dernières années du siècle ; la période retenue, commune aux deux stations, est donc 1901-1990. Afin de comparer les anomalies de ces deux stations dont les hauteurs de précipitations mensuelles et annuelles sont fort différentes (en 1901-1990 : Salta, environ 700 mm ; Mendoza, environ 200 $\mathrm{mm}$ ), les valeurs ont été exprimées en anomalies standardisées (écart à la moyenne rapporté à l'écart-type de la série).

De tels événements se sont déjà produits autrefois, et d'autres successions d'années "pluvieuses" ont déjà affecté ces deux stations, par exemple entre 1917 et 1922. Cependant, la série pluvieuse des années 70 et 80 a été la plus longue du vingtième siècle. À ces années très pluvieuses ont correspondu des années très sèches dans le Sahel ouest-africain (Moron, 1994), avec un léger décalage 


\begin{tabular}{|c|c|c|c|c|c|c|c|c|c|c|c|}
\hline Années & \begin{tabular}{|l|} 
Mendoza \\
\end{tabular} & Salta & EIISO & Années & Mendoza & Salta & EIISO & Années & \begin{tabular}{|l|} 
Mencloza \\
\end{tabular} & Salta & EIISO \\
\hline $1901-1902$ & - & $\mathrm{MQ}$ & 11 & $1931-1932$ & - & + & II & $1961-1962$ & - & - & 11 \\
\hline $1902-1903$ & - & - & $\mathrm{C}$ & $1932-1933$ & - & + & TII & $1962-1963$ & + & + & TII \\
\hline $1903-1904$ & + & - & $\mathbf{F}$ & $1933-1934$ & - & - & $\pi$ & $1963-1964$ & - & + & C \\
\hline $1904-1905$ & + & - & C & $1934-1935$ & - & - & TII & $1964-1965$ & + & - & $\mathbf{F}$ \\
\hline $1905-1906$ & + & - & C & $1935-1936$ & - & + & TII & $1965-1966$ & - & - & C \\
\hline $1906-1907$ & + & + & $F$ & $1936-1937$ & $=$ & - & TII & $1966-1967$ & + & - & $\pi$ \\
\hline $1907-1908$ & - & - & TII & 1937-1938 & - & + & $\pi$ & $1967-1968$ & - & - & $\mathbf{F}$ \\
\hline $1908-1909$ & - & - & $\mathbf{F}$ & $1938-1939$ & - & - & $F$ & $1968-1969$ & - & + & TII \\
\hline 1909-1910 & + & - & $F$ & $1939-1940$ & + & - & TI & $1969-1970$ & + & + & C \\
\hline $1910-1911$ & + & $\mathrm{MQ}$ & $\mathbf{F}$ & $1940-1941$ & + & $=$ & $\mathrm{C}$ & $1970-1971$ & - & - & $F$ \\
\hline $1911-1912$ & + & + & C & $1941-1942$ & - & - & TI & $1971-1972$ & - & $=$ & $\mathbf{F}$ \\
\hline $1912-1913$ & - & + & TII & $1942-1943$ & - & + & $F$ & $1972-1973$ & + & + & C \\
\hline $1913-1914$ & - & - & C & $1943-1944$ & + & - & $\pi$ & $1973-1974$ & $+t$ & + & $\mathbf{F}$ \\
\hline $1914-1915$ & - & - & TI & 1944-1945 & +4 & - & $F$ & $1974-1975$ & + & - & $\pi$ \\
\hline $1915-1916$ & - & - & TI & $1945-1946$ & + & - & TII & $1975-1976$ & - & + & $F$ \\
\hline $1916-1917$ & + & - & $F$ & $1946-1947$ & - & + & $\pi$ & $1976-1977$ & - & - & $\mathrm{C}$ \\
\hline $1917-1918$ & + & ++ & TII & $1947-1948$ & - & - & TII & $1977-1978$ & +4 & + & TII \\
\hline $1918-1919$ & + & - & C & 1948-1948 & + & + & $\pi$ & $1978-1979$ & + & + & $\pi$ \\
\hline $1919-1920$ & + & +4 & TII & $1949-1950$ & - & + & $F$ & $1979-1980$ & + & + & TII \\
\hline $1920-1921$ & + & + & TII & $1950-1951$ & - & - & TII & $1980-1981$ & + & + & TII \\
\hline $1921-1922$ & + & + & TII & $1951-1952$ & + & + & $\mathrm{C}$ & $1981-1982$ & + & - & TII \\
\hline $1922-1923$ & - & + & $F$ & $1952-1953$ & - & - & TI & $1982-1983$ & + & - & C \\
\hline $1923-1924$ & - & - & TII & $1953-1954$ & - & + & TII & 1983-1984 & $+4+$ & + & TII \\
\hline $1924-1925$ & - & - & $F$ & $1954-1955$ & - & - & $F$ & $1984-1985$ & + & + & TII \\
\hline $1925-1926$ & + & - & c & 1955-1956 & - & - & $F$ & $1985-1986$ & + & - & TII \\
\hline $1926-1927$ & + & +4 & TII & $1956-1957$ & - & - & $F$ & $1986-1987$ & + & + & C \\
\hline $1927-1928$ & - & - & TI & $1957-1958$ & + & - & C & $1987-1988$ & + & + & C \\
\hline $1928-1929$ & - & + & $\pi$ & $1958-1959$ & + & + & TII & $1988-1989$ & - & + & $F$ \\
\hline $1929-1930$ & + & - & $\mathrm{C}$ & 1959-1960 & $\mathrm{MQ}$ & + & $\pi$ & $1989-1990$ & +4 & + & $\pi$ \\
\hline $1930-1931$ & + & - & $\mathrm{C}$ & $1960-1961$ & - & - & $\pi$ & $1990-1991$ & $\mathrm{MQ}$ & + & TII \\
\hline
\end{tabular}

Tableau 5 : Phases de l'oscillation australe et anomalies pluviométriques durant les mois les plus arrosés (DJFM) à Mendoza (observatoire) et à Salta, de 1901 à 1990. Les données ont été exprimées en anomalies standardisées. Southern Oscillation phases and precipitation anomalies during the rainiest months (DJFM) at Mendoza (observatory) and Salta, between 1901 and 1990. The data is expressed in standardized anomalies.

Anomalies standardisées :

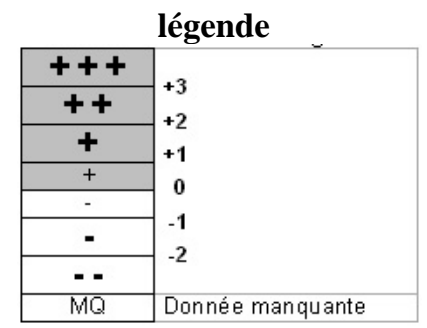

Phases de l'oscillation australe (Année "ENSO" : octobre à septembre de l'année suivante) : anomalies de température de surface de l'Océan Pacifique entre $4^{\circ} \mathrm{S}$ et $4^{\circ} \mathrm{N}$; $90^{\circ} \mathrm{W}$ et $150^{\circ} \mathrm{W}$.

Phase chaude $(\mathrm{C})$ : anomalie égale ou supérieure à $0.5^{\circ} \mathrm{C}$ pendant 6 mois consécutifs ; Phase froide $(\mathrm{F})$ : anomalie égale ou inférieure à $0.5^{\circ} \mathrm{C}$ pendant 6 mois consécutifs ; Phase neutre $(\mathrm{N})$ : valeurs comprises entre -0.5 et $0.5^{\circ} \mathrm{C}$.

Source : Center for Ocean-Atmospheric Prediction Studies (www.coaps.fsu.edu). 
vers l'avant en Argentine. L'opposition de phase pluviométrique entre une partie de l'Amérique du Sud tropicale et subtropicale d'une part et le Sahel d'autre part a déjà été mentionnée par C.K. Folland et al. (1986). Quelques données manquantes à Mendoza n'ont pas permis de réaliser le tableau sur la totalité du siècle ; la période retenue est donc 1901-1990. À Tucumán, la dernière décennie du siècle, malgré une forte variabilité interannuelle, semble avoir conservé des totaux annuels relativement élevés (Prat et Sayago, 2003), de même sur l'ensemble du Nord-Ouest argentin, ainsi pour la plupart des 11 stations dont les séries pluviométriques de la période 1951-2000 sont les plus complètes (tableau 3). Même pour les stations dont le total annuel a diminué entre les périodes 1971-1990 et 1981-2000 (avec les réserves d'usage liées aux données manquantes), le total annuel de la période 1981-2000 demeurait en deuxième place après celui de 1971-1990, sauf à Catamarca et à Santiago del Estero. La réapparition fréquente d'années à saison pluvieuse moins abondamment arrosée, ou même (une année sur deux) anormalement peu arrosée, est cependant une réalité depuis la dernière décennie du vingtième siècle, au moins à Salta durant les années 1991-1992 à 1999-2000 (figure 8).

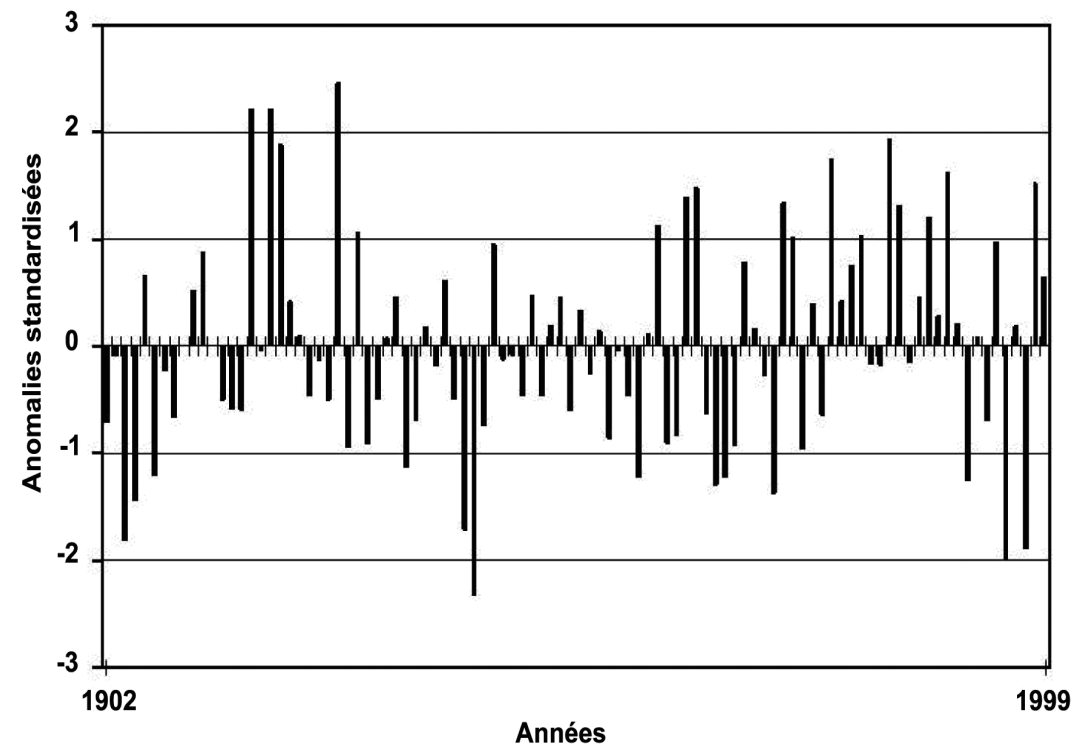

Figure 8 : Chronique des totaux pluviométriques de la période décembre-mars à SALTA, de l'année 1902-1903 à l'année 1999-2000. Precipitation amounts at SALTA for the December - March period, between the year 1902-1903 and the year 1999-2000.

Ces anomalies pluviométriques négatives ont atteint, durant les années 199596 et 1997-98, une intensité inégalée depuis les années 1939-40 et 1940-41. La figure 8 fait aussi bien ressortir les autres années ou séries d'années à anomalies pluviométriques fortement positives observées (tableau 4) durant la première moitié du siècle (par exemple les années 1917-18 à 1922-23), mais aussi la 
durée inégalée, auparavant dans le même siècle, de la période à anomalies pluviométriques positives fréquentes au cours du dernier quart du vingtième siècle : à Salta, à partir de 1972-73 (mais surtout de 1977-78) à 1990-91. Cette période abondamment arrosée succédant, à partir de 1972-1973, à une période relativement sèche (années 50 et 60), confirme pour le Nord-Ouest argentin les observations de J. Ronchail (1996) en Bolivie. Si ces anomalies pluviométriques posent question, la variabilité interne à la période incluant ces anomalies pluviométriques pose aussi question.

La valeur de l'anomalie pluviométrique positive des années 72-90 est cependant variable d'une année à l'autre, et d'une station à l'autre, sans même que l'on puisse a priori déceler un lien avec les événements ENSO (El Niño Southern Oscillation). Ils sont pourtant connectés à des anomalies pluviométriques sur plusieurs espaces d'Amérique du Sud (Ropelewski et Halpert, 1987), y compris en Argentine, mais dans le nord-est du pays (bassin du Paraná : Berri et al., 2002). Afin d'essayer de lever le doute sur cette question, les données ont été soumises à un examen statistique dont les résultats sont présentés dans le tableau 6.

\begin{tabular}{|c|c|c|c|c|c|c|c|c|c|c|c|c|c|}
\hline \multirow{2}{*}{\multicolumn{2}{|c|}{$\begin{array}{r}\text { Période } \rightarrow \\
\text { Marge d'erreur(\%) } \rightarrow\end{array}$}} & \multicolumn{3}{|c|}{$1901-2000$} & \multicolumn{3}{|c|}{ 1901-1990 } & \multicolumn{3}{|c|}{ 1951-2000 } & \multicolumn{3}{|c|}{ 1951-1990 } \\
\hline & & 10 & 5 & 2 & 10 & 5 & 2 & 10 & 5 & 2 & 10 & 5 & 2 \\
\hline \multirow{2}{*}{ SALTA } & Année & 0.18 & 0.18 & 0.18 & 0.08 & 0.08 & 0.08 & I & 1 & 1 & I & 1 & 1 \\
\hline & DJFM & 0.22 & 0.22 & 0.22 & 0.17 & 0.17 & 0.17 & 0.10 & 0.10 & 0.10 & 0.07 & 0.07 & 0.07 \\
\hline \multirow{2}{*}{ MENDOZA } & Année & I & 1 & 1 & -0.13 & -0.13 & -0.13 & 1 & I & 1 & 1 & 1 & I \\
\hline & DJFM & 1 & 1 & 1 & -0.13 & -0.13 & -0.13 & 1 & I & 1 & -0.15 & -0.15 & -0.15 \\
\hline
\end{tabular}

Tableau 6 : Corrélations entre l'indice d'oscillation australe (SOI), les précipitations annuelles et les mois les plus arrosés (DJFM), à Salta et à Mendoza (observatoire) durant le $\mathrm{XX}^{\mathrm{e}}$ siècle. La significativité des coefficients a été examinée au moyen de la table du "r" de Bravais-Pearson (cases grisées : coefficients significatifs). Correlations between the Southern Oscillation Index (SOI), the annual precipitation and the rainiest months (DJFM), at Salta and Mendoza (observatory), during the twentieth century. The significance of the coefficients was examined with the " $r$ "table of Bravais-Pearson (grey boxes : significant coefficients).

La variabilité des précipitations "estivales" a été mise en relation avec les anomalies de température de surface de l'Océan Pacifique tropical (Vuille et al., 2000 ; Garreaud et Aceituno, 2001), en particulier avec les événements liés à l'oscillation australe. L'oscillation australe est un phénomène climatique se traduisant dans l'atmosphère par une variation à intervalles irréguliers (de 3 à 7 ans) du champ de la pression atmosphérique moyenne entre l'ouest et le sudest du Pacifique tropical. On caractérise généralement ce phénomène par un indice exprimant la différence de pression entre Papeete (Tahiti) et Darwin (nord de l'Australie). Cet indice est négatif en période $\underline{E l ~ N i n ̃ o}$ (phase

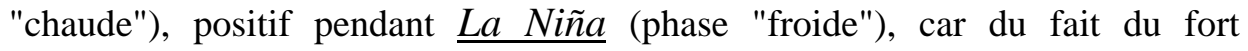
couplage existant entre océan et atmosphère, El Niño et La Niña constituent les 
deux états extrêmes de l'oscillation australe (Météo-France : http://www.meteo.fr/meteonet/decouvr/a-z/html/). Les très mauvaises corrélations obtenues entre les précipitations et l'indice d'oscillation australe (SOI : Southern Oscillation Index) traduisent la complexité du problème de l'analyse de la variabilité pluviométrique dans le Nord-Ouest argentin.

A.R. Bianchi et C.E. Yañez (1992) avaient relevé une tendance significative, mais faible, à une baisse des précipitations durant les années El Niño, à l'aide d'un réseau de 380 stations. M.H. Trauth et al. (2000) ont confirmé cette tendance, avec les mêmes données, mais ont montré qu'elle est soumise à une forte variabilité spatio-temporelle. C'est seulement à l'extrême nord de l'espace étudié (Jujuy) qu'une relation significative entre précipitations et l'ENSO a pu être observée, dans le sens d'une diminution (augmentation) des pluies de novembre à janvier de l'année El Niño (La Niña) en cours (Marwan et al., 2003), en accord avec les résultats obtenus par J. Ronchail plus au nord dans les Andes boliviennes (1994). Plus au sud, cette relation est déjà plus faible à Salta, comme le confirment les résultats présentés dans le tableau 3.

Encore plus au sud, à Tucumán, aucun lien n'a été montré, et les événements ENSO pourraient ne pas influer sur les précipitations dans la partie sud de l'espace étudié, où cette influence est diffuse ou variable dans le temps (Marwan et al., 2003). Selon ces mêmes auteurs (mais déjà observé par P. Xie et P.A Arkin, 1997), la saison pluvieuse est plus abondante durant les épisodes La Niña, en raison du renforcement des alizés, dont l'influence s'accentue en direction du tropique. L'ascendance orographique des masses d'air humide est d'autant plus activée sur le versant oriental des Andes. Inversement, les périodes El Niño sont associées à un affaiblissement des alizés et donc de la saison pluvieuse. La diminution de l'influence des alizés vers le sud, et le passage à la circulation d'ouest extratropicale, contribue à expliquer, pour le reste de l'espace étudié, la difficulté à mettre en évidence un lien entre l'ENSO et les précipitations, compte tenu de la complexité des faits circulatoires. Dans les Andes de Mendoza, ce sont plutôt les précipitations hivernales qui sont corrélées à l'ENSO (anomalies positives en période El Niño : Norte et al., 1998). De plus, seulement 4 années "froides" ont été observées entre 1971-72 et 1990-91 (6 durant les 20 années précédentes), dont 1973-74 a été la seule caractérisée par une anomalie pluviométrique positive significative à Salta et à Mendoza.

Des anomalies pluviométriques positives ont été observées à Salta et à Mendoza durant certaines années succédant à celles de l'événement El Niño (au $\mathrm{XX}^{e}$ siècle : 1919-20, 1926-27, 1973-74, 1977-78, 1983-84). L'excès de précipitations de 1983-1984 a été généralisé sur les Andes boliviennes (Ronchail, 1994). N. Marwan et al. ont observé aussi des réponses pluviométriques opposées après un délai de 8 à 12 mois, mais le mécanisme en cause dans cette relation n'est pas connu. Ils l'expliquent éventuellement par des 
événements La Niña qui peuvent succéder aux événements El Niño, mais, dans le cas des anomalies pluviométriques de Salta et Mendoza, ce cas précis ne s'est produit qu'entre 1972-73 (année El Niño) et 1973-74 (anomalie pluviométrique en phase "froide"). Notons que l'anomalie pluviométrique positive de 19891990 succède à une année en phase "froide". Chercher des liens entre la variabilité pluviométrique du Nord-Ouest argentin et l'oscillation australe fait donc ressortir davantage encore toute la complexité du problème: la climatologie du "Cône Sud" américain est encore loin d'avoir livré tous ses secrets...

\section{Conclusion}

Dans le Nord-Ouest argentin, les dernières décennies du vingtième siècle ont été caractérisées par une succession prédominante d'années anormalement arrosées. Les séries les plus complètes pour les périodes 1951-2000 et 19012000 font ressortir cette série d'années à anomalies pluviométriques positives durant la période 1971-1990. Le renforcement récent de l'abondance pluviométrique estivale a toutefois été inégalement ressenti selon les stations et n'a pas été significatif au point de modifier, même temporairement, la trame climatique de l'espace étudié. Seules des stations en marge du domaine marqué par l'aridité ont nettement été affectées. L'agriculture et la production agricole, sur de tels espaces de marges semi-arides et arides, sont sensibles à la moindre anomalie. Or, l'augmentation des précipitations depuis les années 1970 a coïncidé, dans le Nord-Ouest de l'Argentine, avec une extension et une intensification des activités agricoles (Salomon et Prat, 2004). L'augmentation des ressources hydriques a favorisé la mise en culture de terres marginales ou l'introduction d'espèces plus exigeantes en eau (Gonzalez Loyarte, 1995). Le retour à des conditions climatiques plus sèches pourrait donc avoir de graves conséquences socio-économiques et écologiques (intensification des processus érosifs : Capitanelli, 1967). Un rapport de l'United States Department of Agriculture (décembre 2003) mentionne une réduction de la production de soja, maïs, tournesol, sorgho et arachides, causée par la sécheresse de l'été 20032004, en particulier dans la province de Córdoba. L'étude de la variabilité pluviométrique des provinces du Nord-Ouest de l'Argentine nécessite donc une grande attention dans le contexte économique actuel de cette région.

\section{Remerciements}

Les auteurs remercient Mme Isabelle Ganzetti-Gemin (COSTEL-LETG / UMR 6554 CNRS) pour son aide apportée à la réalisation des cartes.

\section{Bibliographie}

BERRI G.J., GHIETTO M.A. et GARCÍA N.O., 2002: The influence of ENSO in the flows of the Upper Paraná River of South America over the past 100 years. Journal of Hydrometeorology, 3 (1), 57-65. 
BIANCHI A.R. et YAÑEZ C.E., 1992 : Las precipitaciones en el noroeste Argentino. Instituto Nacional de Tecnología Agropecuaria, Estación Experimental Agropecuaria Salta.

BLÜTHGEN J., 1964 : Allgemeine Klimageographie. Walter de Gruyter \& Co., Berlin, $599 \mathrm{p}$.

BRISSE H., GRANDJOUAN R., DE RUFFRAY P., 1982: Les types de climats annuels, un mode d'expression des gradients climatiques intégrant les variations interannuelles. La Météorologie, 31, 39-81.

CAPITANELLI R., 1967: Climatología de la provincia de Mendoza. Bol. Est. Geogr., UNC, Mendoza, 20 (54-57), 681 p.

ENDLICHER W., 1998 : Stadtklima und Lufthygiene argentinischer Andenrandstädte. Tübinger Geographische Studien, 119, 325-352.

FOLLAND C.K., PALMER T.N., PARKER D.E., 1986: Sahel rainfall and worldwide sea temperature, 1901-1985. Nature, 320, 602-607.

GARREAUD R. et ACEITUNO P., 2001: Interannual rainfall variability over the South American Altiplano. Journal of Climate, 14, 2779-2789.

GONZALEZ LOYARTE M.M., 1995: La diagonale aride argentine : une réalité écologique oscillante. Sécheresse, 6, 35-44.

HANSEN J.W., JONES J.W., MAGRIN G., MEIRA S.G., GUEVARA E.R., TRAVASSO M.I., DÍAZ R.A., MARINO M., HORDIJ H., HARWELL C. et PODESTA G., 1996: ENSO effects on yields and economic returns of wheat, corn, and soybean in Argentina. Actas del VII Congreso Argentino y VII Congreso Latinoamericano e Ibérico de Meteorología, Buenos Aires (Argentina), 89-90.

LAUER W., 1993: Klimatologie. Das Geographische Seminar, Westermann, Braunschweig, 267 p.

MARTONNE E. de, 1935 : Problèmes des régions arides sud-américaines. Annales de Géographie, 247, 1-27.

MARWAN N., TRAUTH M.H., VUILLE M. et KURTHS J., 2003: Comparing modern and pleistocene ENSO-like influences in NW Argentina using nonlinear time series analysis methods. Climate Dynamics, 21, 317-326.

METEO-FRANCE : http://www.meteo.fr/meteonet/decouvr/a-z/html/

MORON V., 1994 : Variabilité des précipitations en Afrique tropicale au nord de l'équateur (1933-1990) et relations avec les températures de surface océanique et la dynamique de l'atmosphère. Thèse de Doctorat, Université de Bourgogne, Dijon, $219 \mathrm{p}$.

NORTE F., SIMONELLI S. et HEREDIA N., 1998 : Impacto del fenomeno ENOS en el regimen hidrometeorologico de Mendoza, Argentina. Programma Regional de Meteorología (IANIGLA-CONICET), Gobierno de Mendoza, 10 p.

PLANCHON O., 1997: Les climats maritimes dans le Monde. Thèse de Doctorat, Presses Universitaires du Septentrion, Villeneuve d'Ascq, 233 p. 
PLANCHON O., 2003 : Transitions entre climats tropicaux et tempérés en Amérique du Sud : essai de régionalisation climatique. Cahiers d'Outre-Mer, 223, 259-280.

PRAT M.C. et SAYAGO J.M., 2003 : Risques naturels, actions anthropiques et enjeux à San Miguel de Tucumán. Cahiers d'Outre-Mer, 223, 301-326.

PROHASKA F., 1976 : The climate of Argentina, Paraguay and Uruguay. In : Climates of Central and South America, World Survey of Climatology. Elsevier Scientific Publishing Company, 12 (2), 13-112.

RONCHAIL J., 1994 : La variabilité des précipitations dans les Andes de Bolivie et la phase négative de l'ENSO. Publications de l'Association Internationale de Climatologie, 7, 455-460.

RONCHAIL J., 1996 : Variabilité pluridécennale des précipitations en Bolivie. Essai de mise en relation avec les températures de surface océaniques de l'Atlantique extratropical. Publications de l'Association Internationale de Climatologie, 9, 504511.

ROPELEWSKI C.F. et HALPERT M.S., 1987: Global and regional scale precipitation patterns associated with the El Niño / Southern Oscillation. Monthly Weather Review, 115, 1606-1626.

SALOMON J.N. et PRAT M.C., 2004 : Le piémont andin argentin : environnement, risques et enjeux. Travaux du L.G.P.A., Université Bordeaux-3, 241 p.

TRAUTH M.H., ALONSO R.A., HASELTON K., HERMANNS R. et STRECKER M.R., 2000 : Climate change and mass movements in the northwest Argentine Andes. Earth Planet Science Letter, 179, 243-256.

VUILLE M., BRADLEY R.S. et KEIMIG F., 2000: Interannual climate variability in the Central Andes and its relation to tropical Pacific and Atlantic forcing. Journal of Geophysical Research, 105, 12447-12460.

UNITED STATES DEPARTMENT OF AGRICULTURE, 2003: World Agricultural Production : Drought delays summer-crop planting. Foreign Agricultural Service, Circular Series WAP 12-03, 25 p.

XIE P. et ARKIN P.A., 1997: Global precipitation : a 17-year monthly analysis based on gauge observations, satellite estimales, and numerical model outputs. Bulletin American Meteorological Society, 78, 2539-2558.

ZHOU J. et LAU K.M., 1998: Does a monsoon climate exist over South America ? Journal of Climate, 11, 1020-1040.

\section{Sources des données :}

Center for Ocean-Atmospheric Prediction Studies (www.coaps.fsu.edu).

GHCN (Global Historical Climatology Network). Fichier de données développé par : CDIAC / ORNL (Carbon Dioxide Information Analysis Center / Oak Ridge National Laboratory) et NCDC (National Climatic Data Center). http://cdiac.esd.ornl.gov/ghcn/ghcn.html. 